\title{
Exoplanets, the emergence of a new pathway to understand the origin of life \\ DIDIER QUELOZ
}

University of Geneva

Presenting Author: dq212@cam.ac.uk

The origin of life is one of the grandest outstanding questions in the modern sciences.

The wealth and diversity of planetary systems that have now been detected orbiting stars (exoplanets) present an opportunity of historical perspectives. It prompts an irresistible call to look for signs of life on these new worlds as a way to explore our own origins. Over the last decade this distant goal has moved from the audacious to the achievable, creating a new era of empiricism in the quest for understanding the origins and ubiquity of life that unites Chemistry, Biology, Astrophysics, and the Earth Sciences.

Main challenges and recent progresses from the astrophysical perspectives will be presented during this conference as well as the emergence of a new paradigm for the origins of life on Earth. 